PHYSICAL REVIEW D 88, 109903(E) (2013)

\title{
Erratum: Modeling the horizon-absorbed gravitational flux for equatorial-circular orbits in Kerr spacetime [Phys. Rev. D 88, 044001 (2013)]
}

Andrea Taracchini, Alessandra Buonanno, Scott A. Hughes, and Gaurav Khanna

(Received 24 October 2013; published 5 November 2013)

DOI: 10.1103/PhysRevD.88.109903

PACS numbers: 04.25.D-, 04.25.dg, 04.25.Nx, 04.30.-w, 99.10.Cd

Equation (11) of this paper should be given by

$$
\begin{aligned}
\left|c_{\ell m}\right|^{2}= & {\left[(\lambda+2)^{2}+4 m q M \omega_{m}-4 q^{2} M^{2} \omega_{m}^{2}\right]\left(\lambda^{2}+36 m q M \omega_{m}-36 q^{2} M^{2} \omega_{m}^{2}\right) } \\
& +(2 \lambda+3)\left(96 q^{2} M^{2} \omega_{m}^{2}-48 m q M \omega_{m}\right)+144 M^{2} \omega_{m}^{2}\left(1-q^{2}\right) .
\end{aligned}
$$

The factor $4 m q M \omega_{m}$ in the first square bracketed expression is missing the factor $m$ in the published version of this paper. This equation is correctly implemented in the code used for the work reported in this paper. 